OPEN ACCESS

Edited by:

Maria Teresa Mascellino,

Sapienza University of Rome, Italy

Reviewed by:

Ana Paula D'Alincourt Carvalho-Assef,

Oswaldo Cruz Foundation

(Fiocruz), Brazil

Fupin $\mathrm{Hu}$,

Fudan University, China

${ }^{*}$ Correspondence:

Guolin Hong

xmhg/9899@sina.com

Xianming Liang

178292958@qq.com

tThese authors have contributed equally to this work

Specialty section:

This article was submitted to

Clinical Microbiology,

a section of the journal

Frontiers in Cellular and Infection

Microbiology

Received: 24 March 2020

Accepted: 28 July 2020

Published: 14 September 2020

Citation:

Fang L, Xu H, Ren X, Li X, MaX Zhou H, Hong G and Liang X (2020)

Epidemiology and Risk Factors for

Carbapenem-Resistant

Klebsiella Pneumoniae and

Subsequent MALDI-TOFMS as a Tool

to Cluster KPC-2-Producing

Klebsiella Pneumoniae, a

Retrospective Study.

Front. Cell. Infect. Microbiol. 10:462.

doi: 10.3389/fcimb.2020.00462

\section{Epidemiology and Risk Factors for} Carbapenem-Resistant Klebsiella Pneumoniae and Subsequent MALDI-TOF MS as a Tool to Cluster KPC-2-Producing Klebsiella Pneumoniae, a Retrospective Study

\author{
Lili Fang ${ }^{1,2,3+}$, Heping X ${ }^{1,2,3+}$, Xiaoying Ren ${ }^{1,2,3+}$, Xun $\mathrm{Li}^{1,2,3}$, Xiaobo Ma ${ }^{1,2,3}$, Haijian Zhou ${ }^{4,5}$, \\ Guolin Hong ${ }^{1,2,3 *}$ and Xianming Liang ${ }^{6,7 *}$

\begin{abstract}
${ }^{1}$ Department of Clinical Laboratory, The First Affiliated Hospital, School of Medicine, Xiamen University, Xiamen, China, ${ }^{2}$ Xiamen Key Laboratory of Genetic Testing, Xiamen, China, ${ }^{3}$ School of Public Health, Xiamen University, Xiamen, China, ${ }^{4}$ State Key Laboratory for Infectious Disease Prevention and Control, Chinese Center for Disease Control and Prevention, National Institute for Communicable Disease Control and Prevention, Beijing, China, ${ }^{5}$ Collaborative Innovation Center for Diagnosis and Treatment of Infectious Diseases, Hangzhou, China, ${ }^{6}$ Center of Clinical Laboratory, School of Medicine, Zhongshan Hospital, Xiamen University, Xiamen, China, ${ }^{7}$ Institute of Infectious Disease, School of Medicine, Xiamen University, Xiamen, China
\end{abstract}

Background: Carbapenem-resistant Klebsiella pneumoniae (CRKP) appeared recently and now presents a particularly critical problem to hospitalized patients worldwide. We aim to investigate the epidemiology and the risk factors for CRKP colonization and infections, and to evaluate the application performance of MALDI-TOF MS in clustering CRKP.

Results: CRKP colonization and infections incidence was $2.7(35 / 1,319,427)$ per 100,000 patient-days. Inpatients in CRKP group had higher medical expense than CSKP group. Inpatients with underlying conditions, particularly with pulmonary diseases, and with antimicrobial use prior to culture within 30 days, especially with carbapenem use, were risk factors for CRKP acquisition. All CRKP isolates were detected producing KPC-2. The MALDI-TOF MS system and PFGE system provided similar results, with a good concordance between the two methods (adjusted Rand's coefficient, 0.846) and a high probability of MALDI-TOF MS to predict PFGE results (Wallace coefficient, 0.908).

Conclusions: Underlying conditions, particularly pulmonary diseases, and antimicrobial use prior to culture within 30 days, especially carbapenem use, are risk factors for CRKP acquisition. Bla $\mathrm{A}_{K C-2}$ is the mainstream gene of CRKP in our geographic area of analysis. As only simple sample preparation is needed and the results can be obtained in a short time, MALDI-TOF MS may be considered a probable alternative to PFGE in clustering KPC-2-producing CRKP.

Keywords: carbapenems, resistance, Klebsiella pneumonia, carbapenem-resistant Klebsiella pneumonia, MALDI-TOF MS 


\section{BACKGROUND}

Carbapenems are widely used due to their broad spectrum of activity. Nevertheless, carbapenem-resistant Klebsiella pneumoniae (CRKP) appeared and now presents a particularly critical problem to hospitalized patients worldwide (Yigit et al., 2001; Canton et al., 2012; McConville et al., 2017; Asai et al., 2018). The limited clinical options often make anti-infective therapy extremely difficult and also cause an extra financial burden on patients. Thus, it is necessary to identify the risk factors to prevent CRKP colonization and infections.

Molecular typing of bacterial isolates is the key strategy to identify clusters that are due to the transmission of clonal strains. Multilocus sequence typing (MLST), the repetitive sequence-based PCR Diversi Lab system and pulsed-field gel electrophoresis (PFGE) are good genotyping approaches, but these techniques remain time-consuming with a substantial cost. Rapid methods for molecular typing in colonization or infections with pathogens can not only provide basis for preventing cloning spread but also timely treatment. Therefore, quick methods that can be easily integrated into the routine work flow and do not cause increased costs are important (Sauget et al., 2017). Recently, matrix-assisted laser desorption ionization-time of flight mass spectrometry (MALDI-TOF MS) has been used as a simple tool for typing in infections with bacteria such as Enterobacter cloacae (Khennouchi et al., 2015). But other researcher do not recommend MALDI-TOF-based typing as a bacterial typing method given the heterogeneity in comparison to genotyping (Sachse et al., 2014). Thus, the application performance of MALDI-TOF MS as a clustering analysis method is still controversial.

Here, we set out to conduct a study for CRKP in Xiamen, a southern area in China, and we considered the following objectives: (1) study the epidemiology and risk factors for CRKP colonization and infections in this area, (2) evaluate the application performance of MALDI-TOF MS in clustering CRKP.

\section{MATERIALS AND METHODS}

\section{Patients and Settings}

With the intent of examining prevalence, the background of the patients and the risk factors of CRKP acquisition (colonization and infection), we conducted a case-controlled study. A retrospective epidemiologic surveillance study of CRKP colonization and infections was conducted within a 1900-bed academic Medical Center in the southern area of China from 1 January 2015 to 31 January 2017. Either CRKP colonization

\footnotetext{
Abbreviations: CRKP, carbapenem-resistant Klebsiella pneumoniae; CSKP, carbapenem-susceptible Klebsiella pneumonia; MALDI-TOF MS, matrix-assisted laser desorption ionization-time of flight mass spectrometry; MLST, multilocus sequence typing; PFGE, pulsed-field gel electrophoresis; PCRs, multiplex polymerase chain reactions; NCBI, National Center of Biotechnology Information; FDA, Food and Drug Administration; MICs, minimum inhibitory concentrations; CLSI, the Clinical and Laboratory Standards Institute; IQR, interquartile range; ICU, intensive care unit; CCI, Charlson comorbidity index; COPD, pulmonary diseases included chronic obstructive pulmonary disease; OR, odds ratio; aOR, adjusted odds ratio; CI, confidence interval; SID, Simpson's index of diversity.
}

or infections cases during the inpatients' stay period in hospital were classified as the case group. Patients who were negative for CRKP but positive for carbapenem-susceptible Klebsiella pneumoniae (CSKP) during their stay in hospital were used as the selection pool for the control group during the same study period. Exclusion criteria were community-acquired colonization and infections, missing key data, screening samples, and subsequent episodes in the same patient. The same exclusion criteria were applied to cases and controls.

CRKP cases were selected by a review of microbiological reports. All identified inpatients were initially eligible to participate, and their medical charts were reviewed. For inpatients with multiple episodes of colonization and infection with CRKP, only data relevant to the first episode were collected and analyzed. A colonization or infection case is defined according to CDC definitions of nosocomial infections (Garner et al., 1988).

The CSKP cases as control group were randomly selected from the same units where the inpatients isolated with CRKP during the study period. Records of the control participants were cross-referenced with microbiology results to ensure that they did not have any CRKP positive cultures. Controls whose records had insufficient information were replaced by other randomly selected controls. For inpatients with multiple episodes of infection with CSKP, only data relevant to the first episode were collected and analyzed. The age ( \pm 2 years) and sexes of the patients were matched to inpatients with CRKP colonization and infections, and the ratio for the CRKP:CSKP group was 1:2. We used age ( \pm 2 years) and sexes as the matching variables because both two are strong confunders and good candidates for direct mathing (Mansournia et al., 2018). We set a ratio of $1: 2$ in this study for two reasons: (1) concern for sufficient numbers in a stratified analysis; and (2) the increase in power given the expected prevalence of exposure among the controls (Hennessy et al., 1999).

Both case and control groups data were collected from a database of hospital infection monitoring system. This database drew information from numerous sources, including patients' electronic health record, laboratory, microbiology, and medication administration records.

For identifying possible risk factors of CRKP colonization and infections, patients' demographic characteristics and medical conditions were collected from the electronic sources mentioned above by comparing the CRKP and CSKP groups.

This study was approved by the local Ethics Committee of The First Affiliated Hospital of Xiamen University and complied with the Declaration of Helsinki (2008). Written and informed consent was obtained from all participants.

\section{Definition of CRKP}

A CRKP case was defined as the first clinical Klebsiella pneumoniae positive culture from inpatient with one or more of the following criteria, minimum inhibitory concentrations (MICs) for meropenem/imipenem $\geq 4 \mathrm{mg} / \mathrm{L}$, MICs for ertapenem $\geq 2 \mathrm{mg} / \mathrm{L}$ according to the CLSI guidelines (CLSI, 2020). 


\section{Microbiological Investigations}

Species identification was performed with the Vitek 2 Compact automatic microbial analyzer (BioMerieux, Marcyl'Etoile, France) and confirmed by matrix-assisted laser desorption/ionization time-of-flight mass spectrometry (MALDI-TOF MS; BioMerieux, Marcy-l'Etoile, France).

\section{Molecular Detection}

Multiplex polymerase chain reactions (PCRs) were used to detect the presence of carbapenemase genes (bla $a_{\mathrm{NDM}}$, $b l a_{\mathrm{KPC}}, b l a_{\mathrm{IMP}}$, and $\left.b l a_{\mathrm{VIM}}\right)$. PCR products were sequenced, and the nucleotide and deduced protein sequences were analyzed with software programs that were available from the National Center of Biotechnology Information (NCBI) website (www.ncbi.nlm.nih.gov).

\section{Antimicrobial Susceptibility Testing}

With regard the antimicrobial susceptibility test, MICs of ceftazidime, cefepime, cefotaxime, ceftriaxone, piperacillin/ tazobactam, meropenem, ertapenem, imipenem, aztreonam, amikacin, gentamycin, tobramycin, ciprofloxacin, levofloxacin, trimethopri-sulfamethoxazole, and tigecycline were determined with the Vitek 2 Compact automatic microbial analyzer (BioMrieux, Marcy-l'Etoile, France) according to the Clinical and Laboratory Standards Institute (CLSI) guidelines. In addition, MICs of ertapenem, imipenem, meropenem and colistinpolymyxin-B were determined using E-test strips (BioMerieux, Marcy-l'Etoile, France) according to the manufacturer's instructions. Ertapenem, imipenem and meropenem MICs were interpreted according to the CLSI guidelines. The interpretive criteria for colistin-polymyxin-B was based on the breakpoints of EUCAST. And the interpretive criteria for tigecycline was based on the breakpoints of the Food and Drug Administration (FDA).

\section{Identification and Clustering of Klebsiella Pneumoniae Using MALDI-TOF MS}

The Klebsiella Pneumoniae isolates were plated on Columbia blood agar (bioMérieux, Marcy l'Étoile, France) and incubated for $18 \mathrm{~h}$ to $24 \mathrm{~h}$ at $37^{\circ} \mathrm{C}$. Isolated colonies of each strain were selected and used for MALDI-TOF MS identification using the MALDI-TOF MS (BioMerieux, Marcy-l'Etoile, France), as previously described (Rodel et al., 2019). The obtained spectra were manually selected in the spectra mode of SARAMIS Premium software (BioMerieux, Marcy-l'Etoile, France). Cluster analysis were performed by spectra compared to each other in SARAMIS RUO database according to the manufacturer's instructions (Vitek MS Plus SARAMIS Premium user manual, BioMerieux, Marcy-l'Etoile, France). Consensus spectra were analyzed with a single link agglomerative clustering algorithm, applying the relative taxonomy analysis tool of SARAMIS premium software to show the resulting dendrogram with differences and similarities in relative terms (percent matching masses). As a standard setting, the mass signal intensity was not considered in the cluster analysis. According to the type assignment, we defined a cut-off value was $>75 \%$ similarity (Meng et al., 2019).

\section{Typing of Klebsiella Pneumoniae Using Pulse-Field Gel Electrophoresis (PFGE)}

The 1 day, standardized PFGE protocol (Han et al., 2013) was used for all CRKP isolates during the study periods. Cell suspensions were placed in polystyrene tubes (Falcon; $12 \times$ $75 \mathrm{~mm}$ ), and their optical densities were adjusted to $3.8-4.0$ by a Densimat photometer (BioMérieux, Marcy l'Etoile, France). Slices of CRKP agarose plugs were digested using $50 \mathrm{U}$ of XbaI (TaKaRa Bio, Dalian, China) per slice for $4 \mathrm{~h}$ at $37^{\circ} \mathrm{C}$, and electrophoresis was performed using a CHEF-DRIII system (Bio-Rad Laboratories, Hercules, CA, USA). Electrophoresis was conducted with a switch time of 6 to $36 \mathrm{~s}$ for $18.5 \mathrm{~h}$, and images were captured using a Gel Doc 2000 system (Bio$\mathrm{Rad}$ ) and converted to TIFF files which were analyzed by BioNumerics version 5.1 software (Applied Maths, Kortrijk, Belgium). A similarity analysis of the PFGE patterns was performed by calculating the Dice coefficients $\left(\mathrm{S}_{\mathrm{D}}\right)$ and clustering was performed using the unweighted-pair group method with average linkages (UPGMA).

\section{Statistical Analysis}

CRKP colonization and infections incidence was reported as the number of CRKP cases per 100,000 hospital patientdays. Descriptive statistics were used to summarize the clinical and epidemiologic characteristics of CRKP colonization and infections. Continuous variables were presented as medians with the range or interquartile range. For categorical variables, the percentage of patients or isolates in each category was calculated. The Chi-square test were used to compare categorical variables. The Mann-Whitney U-test was used to compare continuous variables. To identify risk factors for isolating CRKP, the Chi-square test were performed. Factors showing $p<0.05$, were considered candidate predictors that were significantly related to CRKP isolation and were extracted; following which, multivariate analysis was performed for these factors using the Logistic Regression model. The discriminatory power of each typing method was assessed using Simpson's index of diversity (SID), calculating the probability that two unrelated strains sampled from the test population will be placed into different typing groups (Hunter and Gaston, 1988), and the 95\% confidence intervals (CI) of the SID values were calculated as described previously (Grundmann et al., 2001). The quantitative concordance between typing methods was analyzed by using adjusted Randand Wallace coefficients (Carrico et al., 2006). All analyses were performed using the IBM SPSS statistical software package version 25 (IBM Corp, Armonk, NY, USA).

\section{RESULTS}

\section{Prevalence of CRKP Colonization and Infections}

CRKP colonization and infections incidence during 1 January 2015 and 31 January 2017 was $2.7(35 / 1,319,427)$ per 100,000 patient-days. During 1,319,427 patient-days, we found that 2,875 patients with Enterobacteriaceae isolates were obtained, and 36 patients with CRKP colonization and infections were eligible for 
screening in this study. After application of the exclusion criteria, 35 inpatients were included. Five of 35 patients isolating CRKP had infections. All the five were bloodstream infections, all were cured. The characteristics of the inpatients are shown in Table 1 and included 27 males and 8 females. The median age was 73 years (range $0-91$ years).

\section{Clinical and Microbiological Characteristics in CRKP Inpatients}

We found that $25.7 \%$ (9/35) of inpatients had functional status deterioration seen in Table 1. One patient in ICU died within 30 days of admission that was not due to that of a bloodstream infection, but of multiple organ failure caused by cancer, the same reason as the one patient died in CSKP group. CRKP group patients had higher medical expense than those among CSKP group (as shown) in Table $1(p=0.015)$. With regard the antimicrobial susceptibility test, colistin-polymyxin-B, and tigecycline retained excellent activity, with a susceptibility rate of more than $97 \%$. Trimethopri-sulfamethoxazole remained quite susceptible, with susceptibility rate of $57.1 \%$. All isolates of CRKP were detected producing KPC-2 carbapenemase. Further, no CRKP was detected producing two or more gene types of carbapenemase.

\section{Analysis of Risk Factors for Patients Isolating CRKP}

The results of univariate analysis using the Chi-square test in patients with CRKP are shown in Table 1. Eight parameters were associated with patients isolating CRKP, namely one or more underlying conditions $(p=0.007)$, pulmonary diseases $(p<$ $0.001)$, gastric tube $(p<0.001)$, antifungal agents $(p=0.021)$, one or more antimicrobial use prior to culture within 30 days $(p$ $<0.001)$, carbapenem use $(p<0.001)$, quinolone use $(p<0.001)$ and length of stay $(p<0.001)$.

Multivariate logistic regression analysis was applied to analyze the prognostic significance of these eight factors, revealing that one or more underlying conditions ( $p=0.031$, odds ratio [OR]: $3.991,95 \%$ confidence interval $[\mathrm{CI}]: 1.132-14.068)$, pulmonary diseases $(p=0.007$, odds ratio $[\mathrm{OR}]: 5.293,95 \%$ confidence interval [CI]: 1.590-17.618), one or more antimicrobial use prior to culture within 30 days ( $p=0.009$, odds ratio [OR]: $17.358,95 \%$ confidence interval [CI]: 2.051-146.931) and carbapenem use ( $p$ $=0.018$, odds ratio $[\mathrm{OR}]: 5.118,95 \%$ confidence interval $[\mathrm{CI}]$ : 1.321-19.829) were indeed independent risk factors for patients isolating CRKP. Four different clusters of $35 \mathrm{KPC}$-2-producing CRKP isolates were identified by PFGE and MALDI-TOF MS. Cluster I, II, and III were mainly isolated from geriatrics and respiratory wards. Cluster IV was mainly isolated from pediatrics and icu departments.

\section{Clonal Typing KPC-2 Producing CRKP by PFGE}

The PFGE system identified four different clusters of $35 \mathrm{KPC}$ 2-producing CRKP isolates (Figure 1A). All indistinguishable isolates in four clusters presented an average genomic similarity
TABLE 1 | Comparison with patients' characteristics between CRKP and CSKP groups.

\begin{tabular}{|c|c|c|c|}
\hline Characteristic $^{a}$ & $\begin{array}{l}\text { CRKP group }^{\mathrm{b}} \\
(n=35) n, \%\end{array}$ & $\begin{array}{l}\text { CSKP group }^{\mathrm{b}} \\
(n=70) n, \%\end{array}$ & $p$-value \\
\hline \multicolumn{4}{|c|}{ Health care exposure during prior year } \\
\hline Acute care hospitalization & $5(14.3)$ & $9(12.9)$ & 0.839 \\
\hline Dialysis & $1(2.9)$ & $2(2.9)$ & 1.000 \\
\hline $\begin{array}{l}\text { Resident of a long-term-care } \\
\text { facility }\end{array}$ & $6(17.1)$ & $14(20.0)$ & 0.725 \\
\hline $\begin{array}{l}\text { Transfer to ICU within } 30 \\
\text { days }\end{array}$ & $6(17.1)$ & $5(7.1)$ & 0.115 \\
\hline Receipt of corticosteroids & $4(11.4)$ & $9(12.9)$ & 0.834 \\
\hline \multicolumn{4}{|l|}{ Underlying conditions } \\
\hline $\begin{array}{l}\text { One or more underlying } \\
\text { conditions }\end{array}$ & $17(48.6)$ & $16(22.9)$ & 0.007 \\
\hline Cancer $^{\mathrm{C}}$ & $4(11.4)$ & $14(20.0)$ & 0.272 \\
\hline Diabetes mellitus & $8(22.9)$ & $16(22.9)$ & 1.000 \\
\hline Heart diseases $^{d}$ & $4(11.4)$ & $6(8.6)$ & 0.638 \\
\hline Hypertension & $11(31.4)$ & $29(41.4)$ & 0.320 \\
\hline Liver diseases $^{e}$ & $7(20.0)$ & $14(20.0)$ & 1.000 \\
\hline Neurological diseases ${ }^{\dagger}$ & $5(14.3)$ & $20(28.6)$ & 0.105 \\
\hline Pulmonary diseases 9 & $28(80.0)$ & $25(35.7)$ & $<0.001$ \\
\hline Renal diseases ${ }^{h}$ & $8(22.9)$ & $16(22.9)$ & 1.000 \\
\hline CCI score [Median (IQR)] & $2.0(4.0)$ & $2.0(4.0)$ & 1.000 \\
\hline $\mathrm{CCl} \geq 3$ & $15(42.9)$ & $31(44.3)$ & 0.889 \\
\hline Smoking history & $6(17.1)$ & $4(5.7)$ & 0.060 \\
\hline \multicolumn{4}{|c|}{ Indwelling devices prior to culture } \\
\hline Central venous catheter & $15(42.9)$ & $20(28.6)$ & 0.143 \\
\hline Gastric tube & $24(68.6)$ & $21(30.0)$ & $<0.001$ \\
\hline Tracheal cannula & $5(14.3)$ & $12(17.4)$ & 0.708 \\
\hline Tracheotomy & $10(28.6)$ & $10(14.3)$ & 0.079 \\
\hline Urinary catheter & $20(57.1)$ & $36(51.4)$ & 0.580 \\
\hline \multicolumn{4}{|l|}{ Laboratory findings } \\
\hline \multicolumn{4}{|l|}{ White blood cells $/ \mathrm{mm}^{3}$} \\
\hline Median (IQR) & $12,350(3,900)$ & $9,790(6,853)$ & 0.054 \\
\hline \multicolumn{4}{|l|}{ Subgroup } \\
\hline$<4,000$ & $0(0.0)$ & $6(8.6)$ & 0.074 \\
\hline$>10,000$ & $24(68.6)$ & $39(55.7)$ & 0.205 \\
\hline $\begin{array}{l}\text { C-reactive protein > } 10 \\
\text { mg/liter }\end{array}$ & $16(45.7)$ & $39(55.7)$ & 0.333 \\
\hline \multicolumn{4}{|l|}{ Procalcitonin } \\
\hline 0.5 to $2 \mathrm{ng} / \mathrm{ml}$ & $5(14.3)$ & $20(28.6)$ & 0.105 \\
\hline$>2 \mathrm{ng} / \mathrm{ml}$ & $10(28.6)$ & $18(25.7)$ & 0.755 \\
\hline Use of proton pump inhibitors & $8(22.9)$ & $18(25.7)$ & 0.749 \\
\hline Antifungal agents & $8(22.9)$ & $5(7.1)$ & 0.021 \\
\hline \multicolumn{4}{|l|}{$\begin{array}{l}\text { Antimicrobial use prior to } \\
\text { culture within } 30 \text { days }\end{array}$} \\
\hline $\begin{array}{l}\text { One or more Antimicrobial } \\
\text { uses }\end{array}$ & $34(97.1)$ & $33(47.1)$ & $<0.001$ \\
\hline $\begin{array}{l}\text { Third- or fourth-generation } \\
\text { cephalosporin use }\end{array}$ & $6(17.1)$ & $15(21.4)$ & 0.605 \\
\hline Carbapenem use & $16(45.7)$ & $5(7.1)$ & $<0.001$ \\
\hline Quinolone use & $14(40.0)$ & $6(8.6)$ & $<0.001$ \\
\hline
\end{tabular}


TABLE 1 | Continued

\begin{tabular}{|c|c|c|c|}
\hline Characteristic $^{\mathrm{a}}$ & $\begin{array}{l}\text { CRKP group } \\
(n=35) n, \%\end{array}$ & $\begin{array}{l}\text { CSKP group } \\
(n=70) n, \%\end{array}$ & $p$-value \\
\hline \multicolumn{4}{|c|}{ Specimen isolating Klebsiella pneumoniae } \\
\hline Respiratory specimen & $13(37.1)$ & $31(44.3)$ & 0.484 \\
\hline Urine & $9(25.7)$ & $16(22.9)$ & 0.746 \\
\hline Blood & $6(17.1)$ & $13(18.6)$ & 0.858 \\
\hline Ascites & $2(5.7)$ & $2(2.9)$ & 0.471 \\
\hline Bile & $1(2.9)$ & $3(4.3)$ & 0.718 \\
\hline Skin & $1(2.9)$ & $2(2.9)$ & 1.000 \\
\hline Others & $3(8.6)$ & $3(4.3)$ & 0.372 \\
\hline Length of stay [Median (IQR)] $]^{\mathrm{i}}$ & $34(38.0)$ & $16(20.0)$ & $<0.001$ \\
\hline \multicolumn{4}{|l|}{ Discharge disposition } \\
\hline Recovery & $7(20.0)$ & $13(18.6)$ & \\
\hline Improvement & $18(51.4)$ & $39(55.7)$ & 0.375 \\
\hline $\begin{array}{l}\text { Patients transfer to other } \\
\text { hospital }\end{array}$ & $0(0.0)$ & $1(1.4)$ & \\
\hline $\begin{array}{l}\text { Functional status } \\
\text { deterioration }\end{array}$ & $9(25.7)$ & $16(22.9)$ & \\
\hline In-hospital mortality & $1(2.9)$ & $1(1.4)$ & 0.015 \\
\hline $\begin{array}{l}\text { Medical expense for admission } \\
\text { (Mean } \pm \mathrm{SD}, \mathrm{RMB} \text { ) }\end{array}$ & $\begin{array}{c}107,472.27 \pm \\
110,564.67\end{array}$ & $\begin{array}{c}60,738.59 \pm \\
72,925.18\end{array}$ & \\
\hline
\end{tabular}

CRKP, carbapenem-resistant Klebsiella pneumoniae; CSKP, carbapem-sensitive Klebsiella pneumoniae; $\mathrm{CCl}$, Charlson comorbidity index.

${ }^{a} \mathrm{QQR}$, interquartile range; ICU, intensive care unit.

${ }^{b}$ Data are presented as the number/total number (\%), unless otherwise indicated.

${ }^{c}$ Cancer includes malignancy of the lung, digestive tract, gynecology, hematological system, and neurological system.

${ }^{d}$ Heart diseases include congestive heart failure, coronary heart disease, valve replacement, and congenital heart disease.

e Liver diseases included cirrhosis, hepatitis, liver abscess, hepar adiposum (i.e., fatty liver), and hepatic injury.

${ }^{f}$ Neurological diseases include stroke, transient ischemic attack, cerebral palsy, and meningitis.

gPulmonary diseases included chronic obstructive pulmonary disease (COPD), asthma, interstitial lung disease, history of pneumonia and tuberculosis, emphysema, respiratory failure, and infection.

${ }^{h}$ Renal diseases include azotemia and chronic kidney disease.

iOnly patients admitted to hospital were evaluated.

ratio of $>90.0 \%$. The four clusters were significantly different from each other in the percentage of similarity.

\section{Clustering CRKP Isolates Using MALDI-TOF MS}

All the 35 CRKP isolates were correctly identified at the species level by MALDI-TOF MS. The hierarchical clustering of MALDITOF peak profiles identified four different clusters, substantially interchangeable with those obtained with the PFGE system (Figure 1B). The statistical analysis of the data showed that the PFGE system (Simpson's index, 0.608; 95\% CI, 0.512-0.705) and MALDI-TOF MS system (Simpson's index, 0.640; 95\% CI, 0.5320.748 ) provided similar results, with a good concordance between the two methods (adjusted Rand's coefficient, 0.846) and a high probability of MALDI-TOF MS to predict PFGE results (Wallace coefficient, 0.908).

\section{DISCUSSION}

This present retrospective case-controlled study assessed potential risk factors for the development of colonization and infections by CRKP in hospitalized patients. In this study, it demonstrates that inpatients with one or more underlying conditions, especially pulmonary diseases, and antimicrobial use prior to culture within 30 days, particularly carbapenem use, were risk factors for CRKP acquisition. And four different clusters of KPC-2-producing CRKP isolates were identified. Cluster I, II, and III were mainly isolated from geriatrics and respiratory wards. Patients with underlying conditions, such as pulmonary diseases, often visit an outpatient clinic or transfer from icu to respiratory ward, or transfer between the two, even from one hospital to another hospital, and they are subsequently exposed to additional health care and antimicrobials, which are among the most prominent risks (Gupta et al., 2011). These patients could have poor functional status and severe clinical symptoms, which not only places them at a greater risk of an infection caused by CRKP but also results in higher medical expense. Our study demonstrated that medical expense for admission of CRKP groups were almost double higher than those of CSKP group $(107,472$ vs. $60,739 \mathrm{RMB}, p=0.015)$.

Among the four classes of $\beta$-lactamases defined by the Ambler classification system, the KPC $\beta$-lactamase, in Bush group $2 \mathrm{f}$, belongs to Class A. Yigit et al. (2001) first reported KPC $\beta$ lactamases in Klebsiella pneumoniae strains isolated from a patient in North Carolina in the United States in 2001. After that, the KPC-producing organisms had being reported globally (Villegas et al., 2006; Wiener-Well et al., 2010; Canton et al., 2012; Mojica et al., 2012; Cuzon et al., 2013; Asai et al., 2018; Kim et al., 2018). Since in 2015, Biberg et al. (2015) reported KPC-2producing Klebsiella pneumoniae in the Midwest region of Brazil, the rapid increase and dissemination of KPC-2, the primary type of $\beta$-lactamases, in CRKP from many areas, has become a significant public health challenge in the whole word (Gaiarsa et al., 2015). In this study, all CRKP isolates were detected with KPC-2 carbapenemase. The bla $a_{\mathrm{KPC}-2}$ is the mainstream gene of CRKP in our geographic area of analysis.

Bacterial typing is an important method to identify the route of pathogen transmission. Currently, the main method for bacterial typing is the time-consuming and expensive molecular biology technique like Pulsed Field Gel Electrophoresis (PFGE) or Multilocus sequence typing (MLST). Nevertheless, with the application to cultured microorganism identification, matrix-assisted laser desorption/ionization timeof-flight mass spectrometry MS (MALDI-TOF MS) presents incomparable advantages. However, as a new method of bacteria clustering, the application performance of mass spectrometry is controversial. Some studies reported that MALDI-TOF MS could be a good bacterial typing method in several kinds of bacteria, such as extended-Spectrum- $\beta$-Lactamaseand armA methyltransferase-producing Enterobacter cloacae clinical isolates, methicillin-resistant Staphylococcus aureus, Acinetobacter baumannii, Serratia marcescens, and Citrobacter freundii (Mencacci et al., 2013; Khennouchi et al., 2015; Steensels et al., 2017; Rodel et al., 2019). But, Jiang et al. (2019) employed 


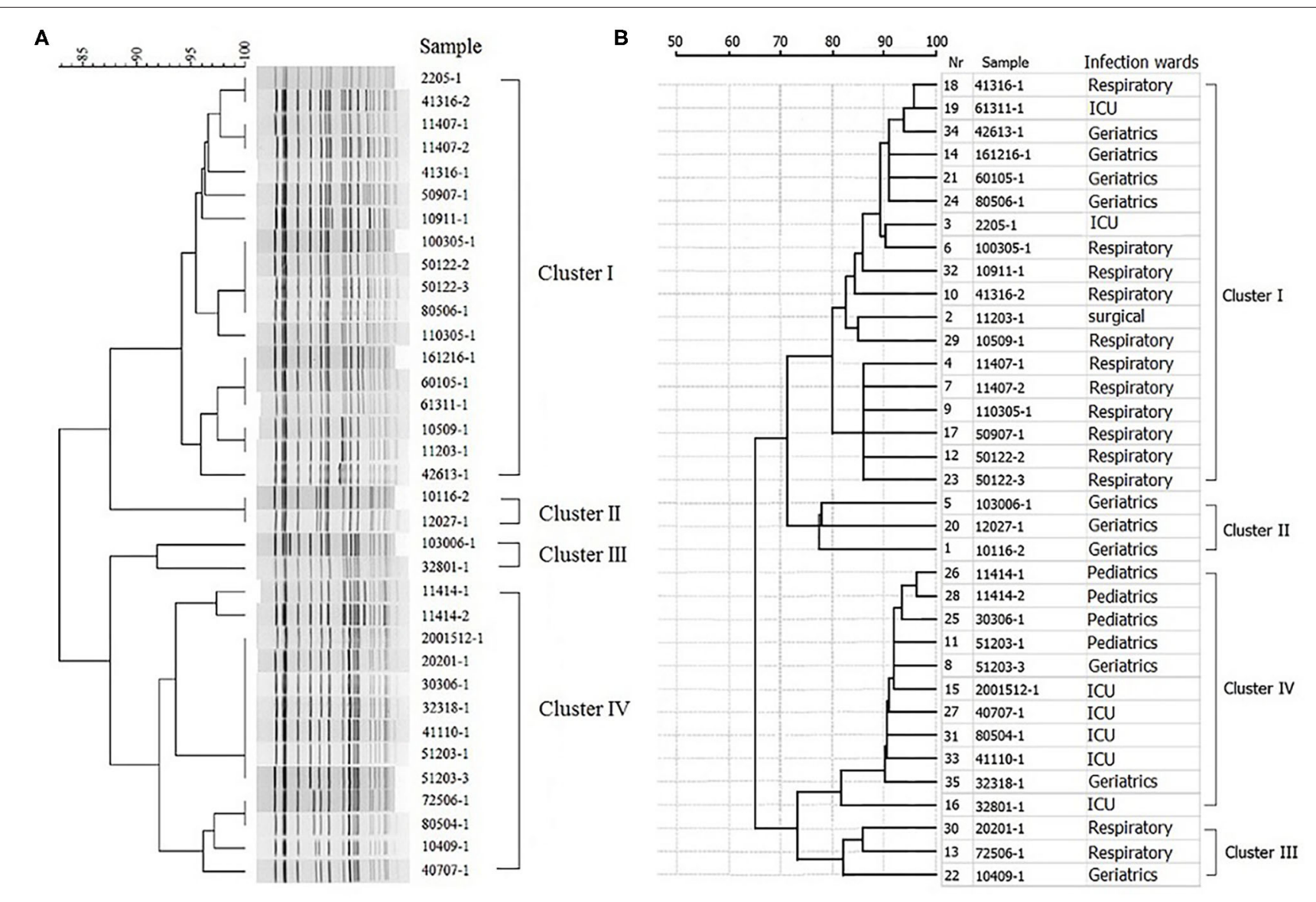

FIGURE 1 | Hierarchical clustering of KPC-2-producing carbapenem-resistant Klebsiella pneumoniae isolates by PFGE and MALDI-TOF MS. (A) Hierarchical cluster analysis provided by PFGE. (B) Hierarchical cluster analysis provided by MALDI-TOF MS.

44 CRKP isolates of 15 STs covering divere carbapenemases and they demonstrated that MALDI-TOF MS had a lower predictive power than PFGE. And Sachse et al. (2014) did not recommend MALDI-TOF-based typing as a bacterial typing method given the heterogeneity in comparison to genotyping.

In this study, all 35 CRKP isolates were correctly identified at the species level by MALDI-TOF MS. The hierarchical clustering of MALDI-TOF peak profiles identified four different clusters, substantially interchangeable with those obtained with the PFGE system. The statistical analysis of the data showed that the PFGE system and MALDI-TOF MS system provided similar results, with a good concordance between the two methods and a high probability of MALDI-TOF MS to predict PFGE results. Since rapid microorganism identification using MADI-TOF MS not only can lead to more effective antimicrobial use and reduced patient care costs (Galar et al., 2012; Tan et al., 2012; Huang et al., 2013; Perez et al., 2013), but also include the high through put, low reagent costs and ease of use, the usage of MALDITOF MS in clustering the CRKP of epidemic KPC-2 type was an agreeable practice and the subsequent clinical application would be meaningful to both hospital infection control and patients. It could be one of the choices to rapidly reveal the routes of transmission of infectious diseases. However, because of the small size of sample, further studies are needed to confirm our observations.

There were three limitations of this study. Firstly, information on the clinical characteristics and outcomes could not be completely acquired because of the limitations that are inherent in a retrospective clinical study. Second, this is a retrospective study with a relatively small study population. Furthermore, this study was a case-controlled design in which the level of risk factors were not equal to the expected level commonly seen in the population.

\section{CONCLUSIONS}

One or more underlying conditions, especially pulmonary diseases, and one or more antimicrobial use prior to culture within 30 days, particularly carbapenem use, are risk factors for CRKP acquisition. The bla $a_{\mathrm{KPC}-2}$ is the mainstream gene of CRKP in our geographic area of analysis. As only simple sample preparation is needed and the results can be obtained in a short time, MALDI-TOF MS may be considered a probable alternative to PFGE in clustering KPC-2-producing CRKP. 


\section{DATA AVAILABILITY STATEMENT}

The raw data supporting the conclusions of this article will be made available by the authors, without undue reservation.

\section{ETHICS STATEMENT}

The studies involving human participants were reviewed and approved by the local Ethics Committee of The First Affiliated Hospital of Xiamen University. Written informed consent to participate in this study was provided by the participants' legal guardian/next of kin. Written informed consent was obtained from the individual(s), and minor(s)' legal guardian/next of kin, for the publication of any potentially identifiable images or data included in this article.

\section{AUTHOR CONTRIBUTIONS}

LF, HX, and XR designed the study. XLi, XM, and HZ analyzed data. LF and XLia drafted the manuscript. GH and XLia contributed to the final version of the manuscript. All authors contributed to the article and approved the submitted version.

\section{REFERENCES}

Asai, N., Sakanashi, D., Suematsu, H., Kato, H., Hagihara, M., Nishiyama, N., et al. (2018). The epidemiology and risk factor of carbapenemresistant enterobacteriaceae colonization and infections: case control study in a single institute in Japan. J. Infect. Chemother. 24, 505-509. doi: 10.1016/j.jiac.2018.02.005

Biberg, C. A., Rodrigues, A. C. S., do Carmo, S. F., Chaves, C. E. V., Gales, A. C., and Chang, M. R. (2015). KPC-2-producing Klebsiella pneumoniae in a hospital in the Midwest region of Brazil. Brazilian J. Microbiol. 46, 501-504. doi: 10.1590/S1517-838246246220140174

Canton, R., Akova, M., Carmeli, Y., Giske, C. G., Glupczynski, Y., Gniadkowski, M., et al. (2012). Rapid evolution and spread of carbapenemases among Enterobacteriaceae in Europe. Clin. Microbiol. Infect. 18, 413-431. doi: 10.1111/j.1469-0691.2012.03821.x

Carrico, J. A., Silva-Costa, C., Melo-Cristino, J., Pinto, F. R., de Lencastre, H., Almeida, J. S., et al. (2006). Illustration of a common framework for relating multiple typing methods by application to macrolide-resistant Streptococcus pyogenes. J. Clin. Microbiol. 44, 2524-2532. doi: 10.1128/JCM.02536-05

CLSI (2020). Performance Standards for Antimicrobial Susceptibility Testing. 30th ed. CLSI Supplement M100. Wayne, PA: Clinical and Laboratory Standards Institute.

Cuzon, G., Naas, T., Correa, A., Quinn, J. P., Villegas, M. V., and Nordmann, P. (2013). Dissemination of the KPC-2 carbapenemase in non-Klebsiella pneumoniae enterobacterial isolates from Colombia. Int. J. Antimicrob. Agents 42, 59-62. doi: 10.1016/j.ijantimicag.201 3.04 .002

Gaiarsa, S., Comandatore, F., Gaibani, P., Corbella, M., Dalla Valle, C., Epis, S., et al. (2015). Genomic epidemiology of Klebsiella pneumoniae in Italy and novel insights into the origin and global evolution of its resistance to carbapenem antibiotics. Antimicrob Agents Chemother. 59, 389-396. doi: 10.1128/AAC.04224-14

Galar, A., Yuste, J. R., Espinosa, M., Guillen-Grima, F., Hernaez-Crespo, S., and Leiva, J. (2012). Clinical and economic impact of rapid reporting of bacterial identification and antimicrobial susceptibility results of the most frequently processed specimen types. Eur. J. Clin. Microbiol. Infect. Dis. 31, 2445-2452. doi: 10.1007/s10096-012-1588-8

\section{FUNDING}

This study was partly supported by grant funding programs from the following esteemed institutions and learned bodies: (1) the Fujian Province Natural Science Foundation of China (grant nos. 2016J01647 and 2016J01643); (2) the Natural Science Foundation of China (grant no. 81772287); (3) the Xiamen important major diseases joint tackle project (grant no. 3502Z20179044).

\section{ACKNOWLEDGMENTS}

We thank Dr. Chaoyang Huang and Dr. Jiaqin Zhang of the Department of Clinical Laboratory, The First Affiliated Hospital, School of Medicine, Xiamen University for thoughtful comments and guidance.

\section{SUPPLEMENTARY MATERIAL}

The Supplementary Material for this article can be found online at: https://www.frontiersin.org/articles/10.3389/fcimb. 2020.00462/full\#supplementary-material

Garner, J. S., Jarvis, W. R., Emori, T. G., Horan, T. C., and Hughes, J. M. (1988). CDC definitions for nosocomial infections, 1988. Am. J. Infect. Control 16, 128-140. doi: 10.1016/0196-6553(88)90053-3

Grundmann, H., Hori, S., and Tanner, G. (2001). Determining confidence intervals when measuring genetic diversity and the discriminatory abilities of typing methods for microorganisms. J. Clin. Microbiol. 39, 4190-4192. doi: 10.1128/JCM.39.11.4190-4192.2001

Gupta, N., Limbago, B. M., Patel, J. B., and Kallen, A. J. (2011). Carbapenemresistant Enterobacteriaceae: epidemiology and prevention. Clin. Infect. Dis. 53, 60-67. doi: 10.1093/cid/cir202

Han, H., Zhou, H. J., Li, H. S., Gao, Y., Lu, Z., Hu, K. X., et al. (2013). Optimization of pulse-field gel electrophoresis for subtyping of Klebsiella pneumoniae. Int. J. Environ. Res. Public Health 10, 2720-2731. doi: 10.3390/ijerph10072720

Hennessy, S., Bilker, W. B., Berlin, J. A., and Strom, B. L. (1999). Factors influencing the optimal control-to-case ratio in matched case-control studies. Am. J. Epidemiol. 149, 195-197. doi: 10.1093/oxfordjournals.aje.a009786

Huang, A. M., Newton, D., Kunapuli, A., Gandhi, T. N., Washer, L. L., Isip, J., et al. (2013). Impact of rapid organism identification via matrix-assisted laser desorption/ionization time-of-flight combined with antimicrobial stewardship team intervention in adult patients with bacteremia and candidemia. Clin. Infect. Dis. 57, 1237-1245. doi: 10.1093/cid/cit498

Hunter, P. R., and Gaston, M. A. (1988). Numerical index of the discriminatory ability of typing systems: an application of Simpson's index of diversity. J. Clin. Microbiol. 26, 2465-2466. doi: 10.1128/JCM.26.11.2465-2466.1988

Jiang, F., Kong, Z., Cheng, C., Kang, H., Gu, B., and Ma, P. (2019). Overestimated discriminatory power of MALDI-TOF mass spectrometry for typing of carbapenem-resistant Klebsiella pneumoniae clones. Epidemiol. Infect. 147:e324. doi: 10.1017/S0950268819002097

Khennouchi, N. C., Loucif, L., Boutefnouchet, N., Allag, H., and Rolain, J. M. (2015). MALDI-TOF MS as a tool to detect a nosocomial outbreak of extended-spectrum-beta-lactamase- and ArmA methyltransferase-producing Enterobacter cloacae clinical isolates in Algeria. Antimicrob Agents Chemother. 59, 6477-6483. doi: 10.1128/AAC.00615-15

Kim, Y. K., Song, S. A., Lee, J. N., Oh, M., Jo, K. M., Kim, H. J., et al. (2018). Clinical factors predicting persistent carriage of Klebsiella pneumoniae carbapenemaseproducing carbapenem-resistant Enterobacteriaceae among patients with known carriage. J. Hosp. Infect. 99, 405-412. doi: 10.1016/j.jhin.2017.10.017 
Mansournia, M. A., Jewell, N. P., and Greenland, S. (2018). Case-control matching: effects, misconceptions, and recommendations. Eur. J. Epidemiol. 33, 5-14. doi: 10.1007/s10654-017-0325-0

McConville, T. H., Sullivan, S. B., Gomez-Simmonds, A., Whittier, S., and Uhlemann, A. C. (2017). Carbapenem-resistant Enterobacteriaceae colonization (CRE) and subsequent risk of infection and 90-day mortality in critically ill patients, an observational study. PLOS ONE 12:e186195. doi: 10.1371/journal.pone.0186195

Mencacci, A., Monari, C., Leli, C., Merlini, L., De Carolis, E., Vella, A., et al. (2013). Typing of nosocomial outbreaks of Acinetobacter baumannii by use of matrixassisted laser desorption ionization-time of flight mass spectrometry. J. Clin. Microbiol. 51, 603-606. doi: 10.1128/JCM.01811-12

Meng, X., Yang, J., Duan, J., Liu, S., Huang, X., Wen, X., et al. (2019). Assessing molecular epidemiology of carbapenem-resistant Klebsiella pneumoniae (CRKP) with MLST and MALDI-TOF in central China. Sci. Rep. 9:2271. doi: 10.1038/s41598-018-38295-8

Mojica, M. F., Correa, A., Vargas, D. A., Maya, J. J., Montealegre, M. C., Rojas, L. J., et al. (2012). Molecular correlates of the spread of KPC-producing Enterobacteriaceae in Colombia. Int. J. Antimicrob. Agents 40, 277-279. doi: 10.1016/j.ijantimicag.2012.05.006

Perez, K. K., Olsen, R. J., Musick, W. L., Cernoch, P. L., Davis, J. R., Land, G. A., et al. (2013). Integrating rapid pathogen identification and antimicrobial stewardship significantly decreases hospital costs. Arch. Pathol. Lab. Med. 137, 1247-1254. doi: 10.5858/arpa.2012-0651-OA

Rodel, J., Mellmann, A., Stein, C., Alexi, M., Kipp, F., Edel, B., et al. (2019). Use of MALDI-TOF mass spectrometry to detect nosocomial outbreaks of Serratia marcescens and Citrobacter freundii. Eur. J. Clin. Microbiol. Infect. Dis. 38, 581-591. doi: 10.1007/s10096-018-03462-2

Sachse, S., Bresan, S., Erhard, M., Edel, B., Pfister, W., Saupe, A., et al. (2014). Comparison of multilocus sequence typing, RAPD, and MALDI-TOF mass spectrometry for typing of $\beta$-lactam-resistant Klebsiella pneumoniae strains. Diagn. Microbiol. Infect. Dis. 80, 267-271. doi: 10.1016/j.diagmicrobio.2014.09.005

Sauget, M., Valot, B., Bertrand, X., and Hocquet, D. (2017). Can MALDI-TOF mass spectrometry reasonably type bacteria? Trends Microbiol. 25, 447-455. doi: 10.1016/j.tim.2016.12.006
Steensels, D., Deplano, A., Denis, O., Simon, A., and Verroken, A. (2017). MALDITOF MS typing of a nosocomial methicillin-resistant Staphylococcus aureus outbreak in a neonatal intensive care unit. Acta Clin. Belg. 72, 219-225. doi: 10.1080/17843286.2016.1198521

Tan, K. E., Ellis, B. C., Lee, R., Stamper, P. D., Zhang, S. X., and Carroll, K. C. (2012). Prospective evaluation of a matrix-assisted laser desorption ionization-time of flight mass spectrometry system in a hospital clinical microbiology laboratory for identification of bacteria and yeasts: a bench-by-bench study for assessing the impact on time to identification and cost-effectiveness. J. Clin. Microbiol. 50, 3301-3308. doi: 10.1128/JCM.01405-12

Villegas, M. V., Lolans, K., Correa, A., Suarez, C. J., Lopez, J. A., Vallejo, M., et al. (2006). First detection of the plasmid-mediated class A carbapenemase KPC-2 in clinical isolates of Klebsiella pneumoniae from South America. Antimicrob Agents Chemother. 50, 2880-2882. doi: 10.1128/AAC.0 0186-06

Wiener-Well, Y., Rudensky, B., Yinnon, A. M., Kopuit, P., Schlesinger, Y., Broide, E., et al. (2010). Carriage rate of carbapenem-resistant Klebsiella pneumoniae in hospitalised patients during a national outbreak. J. Hosp. Infect. 74, 344-349. doi: 10.1016/j.jhin.2009.07.022

Yigit, H., Queenan, A. M., Anderson, G. J., Domenech-Sanchez, A. Biddle, J. W., Steward, C. D., et al. (2001). Novel carbapenemhydrolyzing beta-lactamase, KPC-1, from a carbapenem-resistant strain of Klebsiella pneumoniae. Antimicrob Agents Chemother. 45, 1151-1161. doi: 10.1128/AAC.45.4.1151-1161.2001

Conflict of Interest: The authors declare that the research was conducted in the absence of any commercial or financial relationships that could be construed as a potential conflict of interest.

Copyright (C) 2020 Fang, Xu, Ren, Li, Ma, Zhou, Hong and Liang. This is an openaccess article distributed under the terms of the Creative Commons Attribution License (CC BY). The use, distribution or reproduction in other forums is permitted, provided the original author(s) and the copyright owner(s) are credited and that the original publication in this journal is cited, in accordance with accepted academic practice. No use, distribution or reproduction is permitted which does not comply with these terms. 